\title{
Atomic and Electronic Imaging of Oxide Heterostructures
}

\author{
D. A. Muller*,**, L. Fitting*, A. Ohtomo**, N. Nakagawa***, H. Y. Hwang**,*** \\ *School of Applied and Engineering Physics, Cornell University, Ithaca, NY 14853 \\ **Bell Labs, Lucent Technologies, 600 Mountain Ave, Murray Hill, NJ 07974 \\ ***Department of Advanced Materials, University of Tokyo, Kashiwa, Chiba 277-8561, Japan
}

Electron energy loss spectroscopy (EELS) provides direct information on the local electronic structure of a material. In ionic systems, EELS measurements of formal valences give insight and place remarkable constraints on the structure and stability of heterointerfaces and defects. This is of considerable importance to the field of oxide electronics and ferroelectrics, where been the growth of atomically-abrupt heterointerfaces has been a central goal. However, when the interfaces are between polar and nonpolar layers, electrical and atomic abruptness turn out to be mutually incompatible goals. This is the bulk analogy of the surface reconstructions found in polar systems where a material terminated along a bulk polar plane has a net charge and a divergent surface energy. Unlike conventional semiconductors, for multivalent oxides, physical roughness is not the only option - atomically abrupt interfaces are allowed if electrons can be redistributed at lower energy cost than it takes to redistribute ions. These extra electrons are detectable by EELS.

Recent work on $\mathrm{SrTiO}_{3} / \mathrm{LaAlO}_{3}$ [001] interfaces has found that Ti-La interfaces are conducting, but Sr-Al interfaces are insulating. Our EELS studies show that for the Ti-La terminated interface, excess electrons are found on the Ti sites, but not for the Sr-Al termination (where an extra 1/2 hole would be expected theoretically). Instead significant compensating oxygen vacancies are present and no free holes are found, probably explaining the electrical asymmetry. Controlling the interface termination lets us tune between insulator and conductor, trading chemical for electronic roughness[1].

When both sides of the interface contain mixed-valence ions, the interface can be atomicallyabrupt, but electronically diffuse. Under these conditions, interface phases and charge-modulated structures can be constructed [2]. Structures that are thermodynamically unstable in the bulk can be stabilized by their interface energies in thin layers. As the layer thickness is increased, the structure can fault, in an attempt to recover its bulk valence (fig 1). For the $\mathrm{LaTiO}_{3} / \mathrm{SrTiO}_{3}$ however, the stable oxidized La-phase is the pyrochlore $\mathrm{La}_{2} \mathrm{Ti}_{2} \mathrm{O}_{7}$ which has a unit cell of $\sim 4$ La layers along the [001] direction[3]. In thinner structures a new fault, that does not exist in the bulk, develops along the (001) plane in which a single layer of La sites splits into a double layer of a $1 / 2$ occupied Ruddleston-Popper-like fault. This oxidation reduces the electron count on the neighboring Ti sites (Fig 2). While thin layers are stable against oxidation, they also do not have the bulk electron count, so the stable electron count is limited to $\sim 40 \%$ of the bulk value after thermal annealing (Fig 3 ). The unfaulted structure is stable up to $\sim 400 \mathrm{C}$ [4].

[1] N. Nakagawa, H. Y. Hwang, D. A. Muller, Nature Materials 5, doi:10.1038/nmat1569 (2006).

[2] A. Ohtomo, et al., Nature 419, 378 (2002).

[3] A. Ohtomo, et al., Appl. Phys. Lett. 80, 3922 (2002).

[4] This work supported by the EMMA-ONR MURI under Colin Wood and Cornell Center for

Materials Research, an NSF-MRSEC, and NSF-IMR DMR\#0417392 


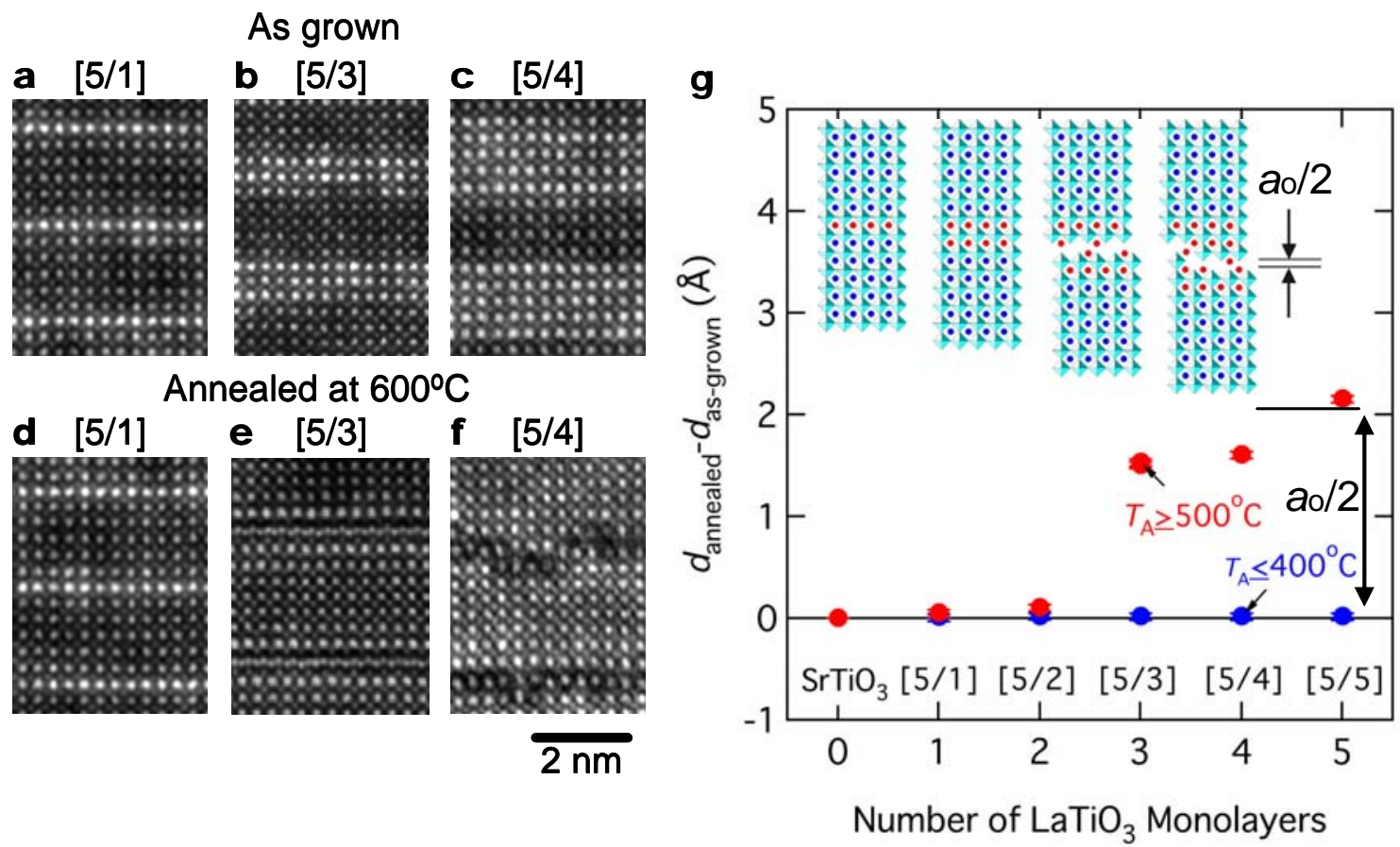

FIG 1. $\left(\mathrm{SrTiO}_{3}\right)_{5} /\left(\mathrm{LaTiO}_{3}\right)_{\mathrm{n}}$ superlattice structures before (a,b,c) and after (d,e,f) annealing in oxygen. A fault opens up in the $\mathrm{LaTiO}_{3}$ layer when more than 2 unit cells are present. (g) shows the expansion of the superlattice period as measured by x-ray diffraction.
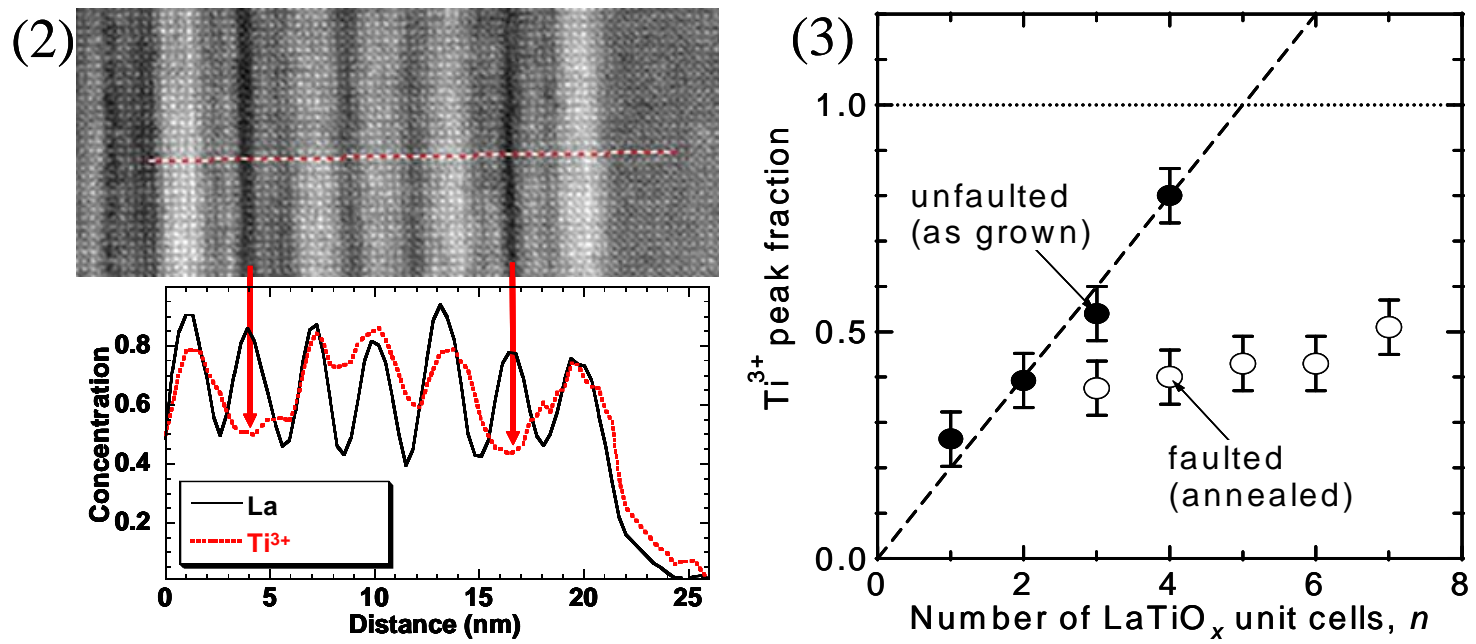

FIG 2. EELS profile across a partially annealed $\left(\mathrm{SrTiO}_{3}\right)_{5} /\left(\mathrm{LaTiO}_{3}\right)_{4}$ superlattice. La modulates the Ti valence (i.e. acts as a dopant) except when a fault occurs (arrows).

FIG 3. Peak $\mathrm{Ti}^{3+}$ fraction for $\left(\mathrm{LaTiO}_{3}\right)_{\mathrm{n}} /\left(\mathrm{SrTiO}_{3}\right)$. Bulk-like $\mathrm{LaTiO}_{3}$ cannot be thinner than 5 layers. Annealing introduces faults in the thicker structures, leading to more fully-oxidized $\mathrm{Ti}$ (ie. $\mathrm{Ti}^{4+}$ )sites. 